Algebraic $\&$ Geometric Topology

Volume 3 (2003) 537-556

ATG

Published: 16 June 2003

\title{
Skein-theoretical derivation of some formulas of Habiro
}

\author{
Gregor Masbaum
}

\begin{abstract}
We use skein theory to compute the coefficients of certain power series considered by Habiro in his theory of $\mathfrak{s l}_{2}$ invariants of integral homology 3 -spheres. Habiro originally derived these formulas using the quantum group $U_{q} \mathfrak{s l}_{2}$. As an application, we give a formula for the colored Jones polynomial of twist knots, generalizing formulas of Habiro and Le for the trefoil and the figure eight knot.
\end{abstract}

AMS Classification 57M25; 57M27

Keywords Colored Jones polynomial, skein theory, twist knots

\section{Introduction}

In a talk at the Mittag-Leffler Institute in May 1999, K. Habiro announced a new approach to computing the colored Jones polynomial of knots and quantum $\mathfrak{s l}_{2}$ invariants of integral homology 3 -spheres. For an exposition, see his paper [H2] (some results are already announced in [H1]). His invariant for homology spheres recovers both the $s l_{2}$ Reshetikhin-Turaev invariants at roots of unity, and Ohtsuki's power series invariants. Later, Habiro and T.Q.T. Le generalized this to all quantum invariants associated to simple Lie algebras.

In the $\mathfrak{s l}_{2}$ case, quantum invariants can be expressed in terms of skein theory, using the Jones polynomial or the Kauffman bracket. Habiro's invariant for homology spheres can be constructed using certain skein elements $\omega=\omega_{+}$and $\omega^{-1}=\omega_{-}$such that circling an even number of strands with $\omega_{+}$(resp. $\left.\omega_{-}\right)$ induces a positive (resp. negative) full twist:<smiles>COCCOC</smiles>

More precisely, $\omega_{+}$and $\omega_{-}$are not skein elements, but infinite sums (i.e. power series) of such. But as long as they encircle an even number of strands (corresponding to a strand colored by an integer-spin representation of $\mathfrak{s l}_{2}$ ), the 
result is well-defined. Also, it makes sense to consider powers of $\omega=\omega_{+}$, and circling an even number of strands with $\omega^{p}$ induces $p$ positive full twists, where $p \in \mathbb{Z}$.

The main purpose of this paper is to give skein-theoretical proofs of Habiro's formulas for $\omega_{+}$and $\omega_{-}$(they are stated already in [H1]) and for $\omega^{p}$ (this formula will appear in [H3]). Habiro's original proofs of these formulas use the quantum group $U_{q} \mathfrak{s l}_{2}$.

This paper is organized as follows. After stating Habiro's formula for $\omega_{+}$and $\omega_{-}$in Section 1, we give a proof using orthogonal polynomials along the lines of [BHMV1] in Section 2. This proof is quite straightforward, although the computations are a little bit more involved than in [BHMV1]. Unfortunately, it seems difficult to use this approach to compute the coefficients of $\omega^{p}$ for $|p| \geq 2$. Therefore, in Section 3 we start afresh using the Kauffman bracket graphical calculus. A first expression for $\omega^{p}$ in Theorem 3.2 is easily obtained, but it is not quite good enough, as an important divisibility property of the coefficients of $\omega^{p}$ is not clear from this formula. This property is then shown in Section 4 by some more skein theory. The final expression for $\omega^{p}$ obtained in Theorem 4.5 is equivalent to Habiro's one from [H3]. (The results of Section 2 are not used here, so that this gives an independent proof in the $p= \pm 1$ case as well.)

To illustrate one use of $\omega^{p}$, we conclude the paper in Section 5 by giving a formula for the colored Jones polynomial of twist knots. This generalizes formulas of Habiro [H1] (see also Le [L1, L2]) for the trefoil and the figure eight knot. (For those two knots, one only needs Habiro's original $\omega_{+}$and $\omega_{-}$.)

Habiro has proved (again using quantum groups) that formulas of this type exist for all knots, but the computation of the coefficients is not easy in general. Formulas of this kind are important for at least two reasons: computing quantities related to the Kashaev-Murakami-Murakami volume conjecture [Ka, MM], and computing Habiro's invariant of the homology sphere obtained by \pm 1 surgery on the knot. For more about this, see Habiro's survey article [H2].

Acknowledgements I got the idea for this work while talking to T.Q.T. Le during his visit to the University of Paris 7 in July 2002. I would like to thank both him and K. Habiro for helpful discussions, and for sending me parts of their forthcoming papers [L2] and [H3]. 


\section{Habiro's formula for $\omega$}

We use the notations of [H1] and of [BHMV1]. In particular, we write

$$
a=A^{2}, \quad\{n\}=a^{n}-a^{-n}, \quad[n]=\frac{a^{n}-a^{-n}}{a-a^{-1}}
$$

and define $\{n\}$ ! and $[n]$ ! in the usual way.

Recall that the Kauffman bracket skein module, $\mathcal{K}(M)$, of an oriented 3manifold $M$ is the free $\mathbb{Z}\left[A^{ \pm}\right]$-module generated by isotopy classes of banded links (= disjointly embedded annuli) in $M$ modulo the submodule generated by the Kauffman relations.

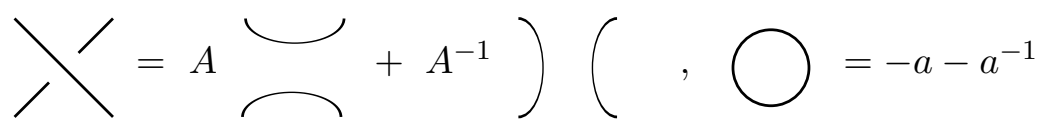

Figure 1: The Kauffman bracket relations. (Recall $a=A^{2}$.)

The Kauffman bracket gives an isomorphism \langle\rangle$: \mathcal{K}\left(S^{3}\right) \stackrel{\approx}{\longrightarrow} \mathbb{Z}\left[A^{ \pm}\right]$. It is normalized so that the bracket of the empty link is 1 .

The skein module of the solid torus $S^{1} \times D^{2}$ is $\mathbb{Z}\left[A^{ \pm}\right][z]$. We denote it by $\mathcal{B}$. Here $z$ is given by the banded link $S^{1} \times J$, where $J$ is a small arc in the interior of $D^{2}$, and $z^{n}$ means $n$ parallel copies of $z$. We define the even part $\mathcal{B}^{\text {ev }}$ of $\mathcal{B}$ to be the submodule generated by the even powers of $z$.

Let $t: \mathcal{B} \rightarrow \mathcal{B}$ denote the twist map induced by a full right handed twist on the solid torus. It is well known (see e.g. [BHMV1]) that there is a basis $\left\{e_{i}\right\}_{i \geq 0}$ of eigenvectors for the twist map. It is defined recursively by

$$
e_{0}=1, \quad e_{1}=z, \quad e_{i}=z e_{i-1}-e_{i-2} .
$$

The eigenvalues are given by

$$
t\left(e_{i}\right)=\mu_{i} e_{i}, \text { where } \mu_{i}=(-1)^{i} A^{i^{2}+2 i} .
$$

Let $\langle$,$\rangle be the \mathbb{Z}\left[A^{ \pm}\right]$-valued bilinear form on $\mathcal{B}$ given by cabling the zeroframed Hopf link and taking the bracket. For $x \in \mathcal{B}$, put $\langle x\rangle=\langle x, 1\rangle$. One has $\left\langle e_{i}\right\rangle=(-1)^{i}[i+1]$.

Moreover, for every $f(z) \in \mathcal{B}$, one has

$$
\left\langle f(z), e_{i}\right\rangle=f\left(\lambda_{i}\right)\left\langle e_{i}\right\rangle \text {, where } \lambda_{i}=-a^{i+1}-a^{-i-1} .
$$


Following Habiro [H1], define

$$
R_{n}=\prod_{i=0}^{n-1}\left(z-\lambda_{2 i}\right), \quad S_{n}=\prod_{i=0}^{n-1}\left(z^{2}-\lambda_{i}^{2}\right) .
$$

The $R_{n}$ form a basis of $\mathcal{B}$, and the $S_{n}$ form a basis of the even part $\mathcal{B}^{e v}$ of $\mathcal{B}$.

By construction, one has $\left\langle R_{n}, e_{2 i}\right\rangle=0$ for $i<n$, and therefore also $\left\langle R_{n}, z^{2 k}\right\rangle=$ 0 for $k<n$. Similarly, one has $\left\langle S_{n}, e_{i}\right\rangle=0$ for $i<n$, and hence also $\left\langle S_{n}, z^{k}\right\rangle=$ 0 for $k<n$. It follows that $\left\langle R_{n}, S_{m}\right\rangle=0$ for $n \neq m$, and for $n=m$ one computes

$$
\left\langle R_{n}, S_{n}\right\rangle=\left\langle R_{n}, e_{2 n}\right\rangle=\left\langle e_{2 n}\right\rangle \prod_{i=0}^{n-1}\left(\lambda_{2 n}-\lambda_{2 i}\right)=(-1)^{n} \frac{\{2 n+1\} !}{\{1\}} .
$$

We are looking for

$$
\omega_{+}=\sum_{n=0}^{\infty} c_{n,+} R_{n}
$$

satisfying (1) for every even $x$, which is equivalent to requiring that

$$
\left\langle\omega_{+}, x\right\rangle=\langle t(x)\rangle
$$

for every $x \in \mathcal{B}^{e v}$. Note that the left hand side of (6) is a finite sum for every $x \in \mathcal{B}^{e v}$.

Theorem 1.1 (Habiro[H1]) Eq. (6) holds for

$$
c_{n,+}=(-1)^{n} \frac{a^{n(n+3) / 2}}{\{n\} !} \text {. }
$$

Let us define $\omega_{-}$to be the conjugate of $\omega_{+}$, where conjugation is defined, as usual, by $\bar{A}=A^{-1}$ and $\bar{z}=z$. Since conjugation corresponds to taking mirror images, we have that

$$
\left\langle\omega_{-}, x\right\rangle=\left\langle t^{-1}(x)\right\rangle
$$

for every even $x$.

Note that $\omega_{-}=\sum_{n=0}^{\infty} c_{n,-} R_{n}$, where

$$
c_{n,-}=\frac{a^{-n(n+3) / 2}}{\{n\} !} .
$$

This follows from (7) since $\overline{R_{n}}=R_{n}$ and $\overline{\{n\}}=-\{n\}$.

Algebraic 83 Geometric Topology, Volume 3 (2003) 
Remark 1.2 The skein element $\omega=\omega_{+}$is related to, but different from, the element often called $\omega$ appearing in the surgery axiom of Topological Quantum Field Theory (see for example [BHMV2]). If we call the latter $\omega^{T Q F T}$, then Equations (6) and (8) would be satisfied by appropriate scalar multiples of $t^{-1}\left(\omega^{T Q F T}\right)$ and $t\left(\omega^{T Q F T}\right)$, respectively; moreover, they would now hold not just for even $x$, but for all $x$. This applies in particular to the $\omega$ of [BHMV1], which was constructed in a similar way as Habiro's $\omega_{-}$(but using polynomials $Q_{n}=\prod_{i=0}^{n-1}\left(z-\lambda_{i}\right)$ in place of the polynomials $\left.R_{n}\right)$.

\section{A proof using orthogonal polynomials}

Habiro's proof of Theorem 1.1 uses the relationship with the quantum group $U_{q} \mathfrak{s l}_{2}$. Here is another proof, using the method of orthogonal polynomials as in [BHMV1].

Testing with the $S_{n}$-basis, we see that (8) holds if and only if

$$
c_{n,-}=\frac{\left\langle t^{-1} S_{n}\right\rangle}{\left\langle R_{n}, S_{n}\right\rangle} \text {. }
$$

Thus, it is clear that an $\omega_{-}$satisfying (8) exists, and to compute its coefficients, we just need to compute $\left\langle t^{-1} S_{n}\right\rangle$.

As in [BHMV1], define another bilinear form $\langle,\rangle_{1}$ by

$$
\langle x, y\rangle_{1}=\langle t(x), t(y)\rangle \text {. }
$$

Define polynomials $\mathscr{R}_{n}$ and $\mathscr{S}_{n}$ by

$$
t\left(\mathscr{R}_{n}\right)=\mu_{n} R_{n}, \quad t\left(\mathscr{S}_{n}\right)=\mu_{2 n} S_{n} .
$$

(The factors $\mu_{n}$ and $\mu_{2 n}$ are included so that $\mathscr{R}_{n}$ and $\mathscr{S}_{n}$ are monic, i.e. have leading coefficient equal to one.)

Again, the $\mathscr{R}_{n}$ form a basis of $\mathcal{B}$, and the $\mathscr{S}_{n}$ form a basis of the even part $\mathcal{B}^{e v}$ of $\mathcal{B}$, since the twist map $t$ preserves $\mathcal{B}^{e v}$. We have $\left\langle\mathscr{R}_{n}, \mathscr{S}_{m}\right\rangle_{1}=0$ for $n \neq m$, and

$$
\left\langle\mathscr{R}_{n}, \mathscr{S}_{n}\right\rangle_{1}=\mu_{n} \mu_{2 n}\left\langle R_{n}, S_{n}\right\rangle .
$$

Note that $\left\langle t^{-1} S_{n}\right\rangle=\mu_{2 n}^{-1}\left\langle\mathscr{S}_{n}\right\rangle$. Thus, we just need to compute $\left\langle\mathscr{S}_{n}\right\rangle$.

Proposition 2.1 The polynomials $\mathscr{S}_{n}$ satisfy a four-term recursion formula

$$
\mathscr{S}_{n+1}=\left(z^{2}-\alpha_{n}\right) \mathscr{S}_{n}-\beta_{n-1} \mathscr{S}_{n-1}-\gamma_{n-2} \mathscr{S}_{n-2}
$$

for certain $\alpha_{n}, \beta_{n-1}, \gamma_{n-2} \in \mathbb{Z}\left[A^{ \pm}\right]$. 
Proof Since $\mathscr{S}_{n}$ is monic of degree $2 n$, we have that $z^{2} \mathscr{S}_{n}-\mathscr{S}_{n+1}$ is a linear combination of the $\mathscr{S}_{k}$ with $k \leq n$. The coefficients can be computed by taking the scalar product with $\mathscr{R}_{k}$. So we just need to show that $\left\langle z^{2} \mathscr{S}_{n}, \mathscr{R}_{k}\right\rangle_{1}=0$ if $k<n-2$.

The point is that multiplication by $z$ is a self-adjoint operator with respect to the bilinear form $\langle,\rangle_{1}$. In other words, one has

$$
\langle z x, y\rangle_{1}=\langle x, z y\rangle_{1}
$$

for all $x, y \in \mathcal{B}$. (This is because $\langle x, y\rangle_{1}=\langle t(x y)\rangle$.) It follows that

$$
\left\langle z^{2} \mathscr{S}_{n}, \mathscr{R}_{k}\right\rangle_{1}=\left\langle\mathscr{S}_{n}, z^{2} \mathscr{R}_{k}\right\rangle_{1}=0 \text { if } k<n-2,
$$

since $\mathscr{R}_{k}$ has degree $k$, and $\mathscr{S}_{n}$ annihilates all polynomials of degree $<n$.

Note that the coefficients in the recursion formula (11) are given by

$$
\alpha_{n}=\frac{\left\langle z^{2} \mathscr{S}_{n}, \mathscr{R}_{n}\right\rangle_{1}}{\left\langle\mathscr{S}_{n}, \mathscr{R}_{n}\right\rangle_{1}}, \beta_{n-1}=\frac{\left\langle z^{2} \mathscr{S}_{n}, \mathscr{R}_{n-1}\right\rangle_{1}}{\left\langle\mathscr{S}_{n-1}, \mathscr{R}_{n-1}\right\rangle_{1}}, \gamma_{n-2}=\frac{\left\langle z^{2} \mathscr{S}_{n}, \mathscr{R}_{n-2}\right\rangle_{1}}{\left\langle\mathscr{S}_{n-2}, \mathscr{R}_{n-2}\right\rangle_{1}}
$$

By convention, if $n<0$ then $\mathscr{R}_{n}, \mathscr{S}_{n}, \alpha_{n}, \beta_{n}, \gamma_{n}$ are all zero.

Proposition 2.2 One has

$$
\begin{aligned}
\alpha_{n} & =2+a^{6 n+4}[3]-a^{2 n} \\
\beta_{n-1} & =\left(a^{4 n+1}+a^{8 n+1}[3]\right)\{2 n\}\{2 n+1\} \\
\gamma_{n-2} & =a^{10 n-4}\{2 n-2\}\{2 n-1\}\{2 n\}\{2 n+1\}
\end{aligned}
$$

Proof The formula for $\gamma_{n-2}$ is the easiest. Let us use the notation $\mathfrak{o}_{\leq n}$ for terms of degree $\leq n$. Since $z^{2} \mathscr{R}_{n-2}=\mathscr{R}_{n}+\mathfrak{o}_{\leq n-1}$, we have

$$
\left\langle z^{2} \mathscr{S}_{n}, \mathscr{R}_{n-2}\right\rangle_{1}=\left\langle\mathscr{S}_{n}, z^{2} \mathscr{R}_{n-2}\right\rangle_{1}=\left\langle\mathscr{S}_{n}, \mathscr{R}_{n}\right\rangle_{1},
$$

and hence formula (15) follows from (12), (10), and (5).

For $\beta_{n-1}$, we need to compute

$$
\left\langle z^{2} \mathscr{S}_{n}, \mathscr{R}_{n-1}\right\rangle_{1}=\left\langle\mathscr{S}_{n}, z^{2} \mathscr{R}_{n-1}\right\rangle_{1}=\mu_{2 n} \mu_{n-1}\left\langle S_{n}, t z^{2} t^{-1} R_{n-1}\right\rangle .
$$

This amounts to computing the coefficient of $R_{n}$ in the expression of $t z^{2} t^{-1} R_{n-1}$ in the $R_{k}$-basis. This coefficient can be computed as follows.

For $n \geq 1$, one has

$$
z^{n}=e_{n}+(n-1) e_{n-2}+\mathfrak{o}_{\leq n-4} .
$$


(This follows by induction from (2).) Thus, for $\varepsilon= \pm 1$, one has

$$
t^{\varepsilon} z^{n}=\mu_{n}^{\varepsilon} z^{n}+(n-1)\left(\mu_{n-2}^{\varepsilon}-\mu_{n}^{\varepsilon}\right) z^{n-2}+\mathfrak{o}_{\leq n-4} .
$$

It follows that

$$
t z^{2} t^{-1} z^{n}=\frac{\mu_{n+2}}{\mu_{n}} z^{n+2}+\left(2-(n+1) \frac{\mu_{n+2}}{\mu_{n}}+(n-1) \frac{\mu_{n}}{\mu_{n-2}}\right) z^{n}+\mathfrak{o}_{\leq n-2} .
$$

Now write

$$
R_{n}=\prod_{i=0}^{n-1}\left(z-\lambda_{2 i}\right)=z^{n}-x_{n-1} z^{n-1}+\mathfrak{o}_{\leq n-2},
$$

where $x_{n-1}=\sum_{i=0}^{n-1} \lambda_{2 i}$. Then (17) gives

$$
t z^{2} t^{-1} R_{n-1}=\frac{\mu_{n+1}}{\mu_{n-1}} R_{n+1}+\left(x_{n} \frac{\mu_{n+1}}{\mu_{n-1}}-x_{n-2} \frac{\mu_{n}}{\mu_{n-2}}\right) R_{n}+\mathfrak{o}_{\leq n-1}
$$

and hence

$$
\left\langle S_{n}, t z^{2} t^{-1} R_{n-1}\right\rangle=\left(x_{n} \frac{\mu_{n+1}}{\mu_{n-1}}-x_{n-2} \frac{\mu_{n}}{\mu_{n-2}}\right)\left\langle S_{n}, R_{n}\right\rangle .
$$

Plugging this into (16), we have

$$
\begin{aligned}
\left\langle z^{2} \mathscr{S}_{n}, \mathscr{R}_{n-1}\right\rangle_{1} & =\mu_{2 n} \mu_{n-1}\left\langle S_{n}, t z^{2} t^{-1} R_{n-1}\right\rangle \\
& =\left(x_{n} \frac{\mu_{n+1}}{\mu_{n}}-x_{n-2} \frac{\mu_{n-1}}{\mu_{n-2}}\right)\left\langle\mathscr{S}_{n}, \mathscr{R}_{n}\right\rangle_{1} \\
& =\left(A^{6 n+1}[3]+A^{-2 n+1}\right)\left\langle\mathscr{S}_{n}, \mathscr{R}_{n}\right\rangle_{1} .
\end{aligned}
$$

Using (12), (10), and (5) as before, this implies formula (14) for $\beta_{n-1}$.

Finally, for $\alpha_{n}$, let us compute

$$
\left\langle z^{2} \mathscr{S}_{n}, \mathscr{R}_{n}\right\rangle_{1}=\mu_{2 n} \mu_{n}\left\langle t z^{2} t^{-1} S_{n}, R_{n}\right\rangle .
$$

This amounts to computing the coefficient of $S_{n}$ in the expression of $t z^{2} t^{-1} S_{n}$ in the $S_{k}$-basis. ${ }^{1}$

The computation is similar to the one above. We write

$$
S_{n}=\prod_{i=0}^{n-1}\left(z^{2}-\lambda_{i}^{2}\right)=z^{2 n}-y_{n-1} z^{2 n-2}+\mathfrak{o}_{\leq 2 n-4},
$$

\footnotetext{
${ }^{1}$ This is easier than computing $\left\langle S_{n}, t z^{2} t^{-1} R_{n}\right\rangle$ by expanding $t z^{2} t^{-1} R_{n}$ in the $R_{k}$ basis, because the latter would require computing the first three terms, and not just the first two terms as in (18) above and also in (20) below.
}

Algebraic $E_{3} \mathcal{G}$ eometric Topology, Volume 3 (2003) 
where $y_{n-1}=\sum_{i=0}^{n-1} \lambda_{i}^{2}$. Then (17) gives

$$
\begin{gathered}
t z^{2} t^{-1} S_{n}= \\
\frac{\mu_{2 n+2}}{\mu_{2 n}} S_{n+1}+\left(2+\left(y_{n}-2 n-1\right) \frac{\mu_{2 n+2}}{\mu_{2 n}}-\left(y_{n-1}-2 n+1\right) \frac{\mu_{2 n}}{\mu_{2 n-2}}\right) S_{n}+\mathfrak{o}_{\leq 2 n-2}
\end{gathered}
$$

and hence

$$
\begin{aligned}
\left\langle t z^{2} t^{-1} S_{n}, R_{n}\right\rangle & =\left(2+\left(y_{n}-2 n-1\right) \frac{\mu_{2 n+2}}{\mu_{2 n}}-\left(y_{n-1}-2 n+1\right) \frac{\mu_{2 n}}{\mu_{2 n-2}}\right)\left\langle S_{n}, R_{n}\right\rangle \\
& =\left(2+a^{6 n+4}[3]-a^{2 n}\right)\left\langle S_{n}, R_{n}\right\rangle .
\end{aligned}
$$

Plugging this into (19), we get

$$
\left\langle z^{2} \mathscr{S}_{n}, \mathscr{R}_{n}\right\rangle_{1}=\left(2+a^{6 n+4}[3]-a^{2 n}\right)\left\langle\mathscr{S}_{n}, \mathscr{R}_{n}\right\rangle_{1},
$$

proving formula (13) for $\alpha_{n}$.

Proof of Habiro's Theorem 1.1 As already observed, we have

$$
c_{n,-}=\frac{\left\langle t^{-1} S_{n}\right\rangle}{\left\langle R_{n}, S_{n}\right\rangle}=\mu_{2 n}^{-1} \frac{\left\langle\mathscr{S}_{n}\right\rangle}{\left\langle R_{n}, S_{n}\right\rangle} .
$$

But $\left\langle\mathscr{S}_{n}\right\rangle$ satisfies the recursion relation

$$
\left\langle\mathscr{S}_{n+1}\right\rangle=\left(\lambda_{0}^{2}-\alpha_{n}\right)\left\langle\mathscr{S}_{n}\right\rangle-\beta_{n-1}\left\langle\mathscr{S}_{n-1}\right\rangle-\gamma_{n-2}\left\langle\mathscr{S}_{n-2}\right\rangle
$$

(since $\left.\langle z\rangle=\lambda_{0}\right)$. It follows that

$$
\left\langle\mathscr{S}_{n}\right\rangle=(-1)^{n} a^{\left(3 n^{2}+n\right) / 2} \frac{\{n+1\}\{n+2\} \cdots\{2 n+1\}}{\{1\}},
$$

since one can check that (22) is true for $n=0,1,2$ and that it solves the recursion (21). This implies Habiro's formula (9) for $c_{n,-}$. Taking conjugates, one then also obtains formula (7) for $c_{n,+}$.

Remark 2.3 Although it might be hard to guess formula (22), once one knows it the recursion relation (21) is easily checked. Observe that $\lambda_{0}^{2}=a^{2}+a^{-2}-2$. Put $q(n)=\left(3 n^{2}+n\right) / 2$. Then (21) is equivalent to

$$
\begin{aligned}
\left(a^{2}+a^{-2}\right. & \left.-a^{6 n+4}[3]+a^{2 n}\right) a^{q(n)}+\left(a^{4 n+1}+a^{8 n+1}[3]\right)\{n\} a^{q(n-1)} \\
& -a^{10 n-4}\{n-1\}\{n\} a^{q(n-2)}=-\frac{\{2 n+2\}\{2 n+3\}}{\{n+1\}} a^{q(n+1)}
\end{aligned}
$$

which is a straightforward computation. 


\section{Graphical calculus and a formula for $\omega^{p}$}

Let us write $\omega=\omega_{+}$and put

$$
\omega^{p}=\sum_{n=0}^{\infty} c_{n, p} R_{n} .
$$

Note that the coefficients $c_{n, p}$ are well-defined (because $R_{n}$ divides $R_{n+1}$ and therefore the coefficients $C_{n, m}^{k}$ in the product expansion $R_{n} R_{m}=\sum_{k} C_{n, m}^{k} R_{k}$ are zero if $n$ or $m$ is bigger than $k$.) We have

$$
\left\langle\omega^{p}, x\right\rangle=\left\langle t^{p}(x)\right\rangle
$$

for every even $x$. (This follows from (6) since circling with $\omega^{p}$ is the same as circling with $p$ parallel copies of $\omega$.) Of course, $c_{n, 1}=c_{n,+}$ and $c_{n,-1}=c_{n,-}$ (it follows from the uniqueness of $\omega_{-}$that $\omega_{-}=\omega^{-1}$ ). The aim of this section is to give a formula for the coefficients $c_{n, p}$ (see Theorem 3.2 below).

We use the extension of the Kauffman bracket to admissibly colored banded trivalent graphs as in [MV]. (Such graphs are sometimes called spin networks; for more background see e.g. [KL] and references therein.) A color is just an integer $\geq 0$. A triple of colors $(a, b, c)$ is admissible if $a+b+c \equiv 0(\bmod 2)$ and $|a-b| \leq c \leq a+b$. Let $D$ be a planar diagram of a banded trivalent graph. An admissible coloring of $D$ is an assignment of colors to the edges of $D$ so that at each vertex, the three colors meeting there form an admissible triple. The Kauffman bracket of $D$ is defined to be the bracket of the expansion of $D$ obtained as follows. The expansion of an edge colored $n$ consists of $n$ parallel strands with a copy of the Jones-Wenzl idempotent $f_{n}$ inserted. (The idempotent $f_{n}$ is characterized by the fact that $x f_{n}=f_{n} x=0$ for every element $x$ of the standard basis of the Temperley-Lieb algebra other than the identity element; here, the standard basis consists of the $(n, n)$-tangle diagrams without crossings and without closed loops.) The expansion of a vertex is defined as in Fig. 2, where the internal colors $i, j, k$ are defined by

$$
i=(b+c-a) / 2, j=(c+a-b) / 2, k=(a+b-c) / 2 .
$$

We have the fusion equation

$$
\frac{a}{b}=\sum_{c} \frac{\langle c\rangle}{\langle a, b, c\rangle} \sum_{b}^{a} \sum_{b}^{a}
$$




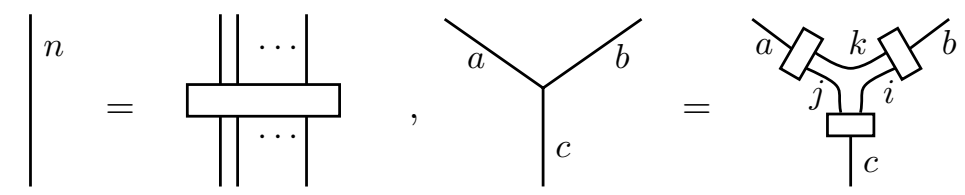

Figure 2: How to expand colored edges and vertices. The boxes stand for appropriate Jones-Wenzl idempotents.

Here the sum is over those colors $c$ so that the triple $(a, b, c)$ is admissible, we have $\langle c\rangle=\left\langle e_{c}\right\rangle=(-1)^{c}[c+1]$, and the trihedron coefficient $\langle a, b, c\rangle$ is (see $[\mathrm{MV}$, Thm. 1]):

$$
\langle a, b, c\rangle=\frac{c}{b}=(-1)^{i+j+k} \frac{[i+j+k+1] ![i] ![j] ![k] !}{[a] ![b] ![c] !}
$$

(here $i, j, k$ are the internal colors as defined in (25)). Note that $\langle n, n, 2 n\rangle=$ $\langle 2 n\rangle$ so that

$$
\frac{n}{n}=\sum_{n}^{n} \sum_{n}^{n}+\cdots
$$

We will need the following lemma.

Lemma 3.1 For $0 \leq k \leq n$, one has

$$
\left.\widehat{n}_{2 k}^{2 n}\right|_{2 n} ^{n}=\left.\frac{([k] !)^{2}}{[2 k] !}\right|^{2 n}
$$

Proof This follows from the formula for the tetrahedron coefficient given in [MV, Thm. 2]. (The sum over $\zeta$ in that formula reduces to just one term.)

The key observation is that

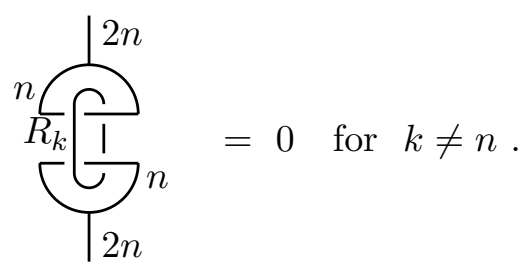

Algebraic 63 Geometric Topology, Volume 3 (2003) 
Indeed, if $k<n$, there are at most $2 k$ vertical strands in the middle and so the result is zero because of the Jones-Wenzl idempotents $f_{2 n}$ at the top and bottom. On the other hand, if $k>n$, the result is zero because $R_{k}$ annihilates all even polynomials in $z$ of degree $<2 k$.

If $k=n$ one finds

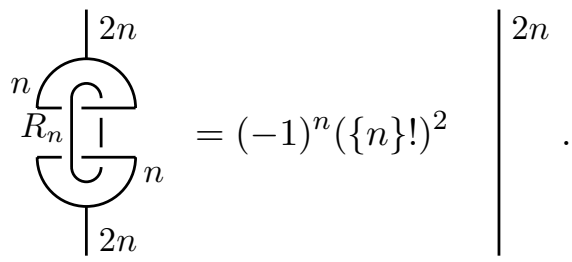

Indeed, applying (28), one has

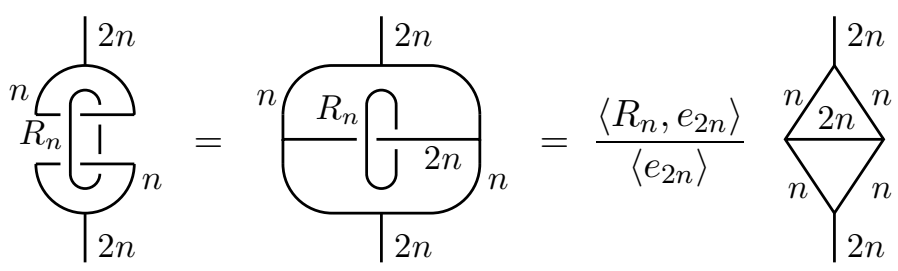

hence (30) follows from (5) and Lemma 3.1.

On the other hand, since circling with $\omega^{p}$ induces $p$ full twists on even numbers of strands (see (24)), we have, using (29), that

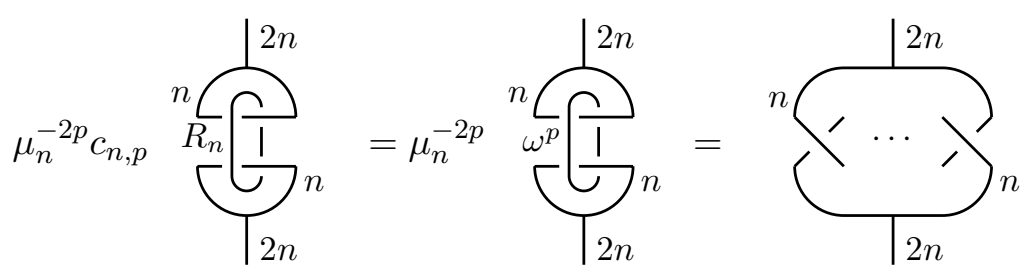

where there are $2 p$ crossings in the last diagram. (See (3) for the twist eigenvalues $\mu_{i}$.) Applying the fusion equation (26), we have

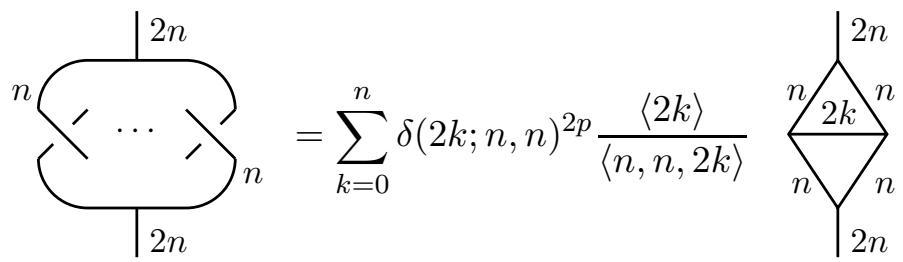

where $\delta(c ; a, b)$ is the half-twist coefficient defined by

$$
\searrow_{a}^{b}=\delta(c ; a, b) \stackrel{b}{\frac{b}{c}} .
$$

Algebraic 83 Geometric Topology, Volume 3 (2003) 
This coefficient is computed in [MV, Thm. 3]. For us, it is enough to know that

$$
\delta(c ; a, b)^{2}=\frac{\mu_{c}}{\mu_{a} \mu_{b}}
$$

which is easy to see. Using (30) and Lemma 3.1, it follows that

$$
\mu_{n}^{-2 p} c_{n, p}(-1)^{n}(\{n\} !)^{2}=\sum_{k=0}^{n} \frac{\mu_{2 k}^{p}}{\mu_{n}^{2 p}} \frac{\langle 2 k\rangle}{\langle n, n, 2 k\rangle} \frac{([k] !)^{2}}{[2 k] !} .
$$

The factors of $\mu_{n}^{-2 p}$ cancel out, and in view of (27), this gives the following result:

Theorem 3.2 The coefficients $c_{n, p}$ of $\omega^{p}$ in (23) are given by

$$
c_{n, p}=\frac{1}{\left(a-a^{-1}\right)^{2 n}} \sum_{k=0}^{n} \frac{(-1)^{k} \mu_{2 k}^{p}[2 k+1]}{[n+k+1] ![n-k] !} .
$$

\section{Another formula for the coefficients of $\omega^{p}$}

Following Habiro, we introduce the polynomials $R_{n}^{\prime}=(\{n\} !)^{-1} R_{n}$ and write

$$
\omega^{p}=\sum_{n=0}^{\infty} c_{n, p}^{\prime} R_{n}^{\prime}
$$

where

$$
c_{n, p}^{\prime}=\{n\} ! c_{n, p} .
$$

The aim of this section is to show that $c_{n, p}^{\prime}$ is a Laurent polynomial, i.e. that $c_{n, p}^{\prime} \in \mathbb{Z}\left[A^{ \pm}\right]$. This fact was shown by Habiro [H3] using the quantum group $U_{q} \mathfrak{s l}_{2}$. Observe that by (9) and (7), we already know this fact for $p= \pm 1$ :

$$
c_{n, 1}^{\prime}=(-1)^{n} a^{n(n+3) / 2}, \quad c_{n,-1}^{\prime}=a^{-n(n+3) / 2} .
$$

(But Formula (33) tells us only that

$$
c_{n, p}^{\prime}=\frac{1}{\left(a-a^{-1}\right)^{n}} \sum_{k=0}^{n}(-1)^{k} \mu_{2 k}^{p}[2 k+1] \frac{[n] !}{[n+k+1] ![n-k] !},
$$

from which it is not clear that $c_{n, p}^{\prime} \in \mathbb{Z}\left[A^{ \pm}\right]$.)

To do so, we will replace Formula (32) in the previous section by Formula (42) below. For this, we need the following two Lemmas. 
Lemma 4.1 We have

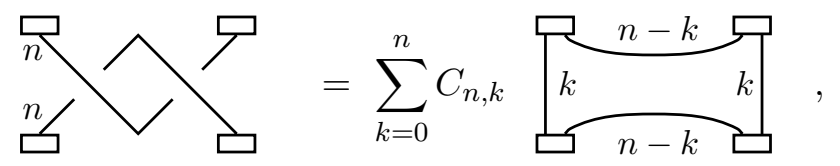

where

$$
C_{n, k}=a^{n(n-k)}\left[\begin{array}{l}
n \\
k
\end{array}\right] \prod_{j=n-k+1}^{n}\left(1-a^{-2 j}\right) .
$$

Here, as usual,

$$
\left[\begin{array}{l}
n \\
k
\end{array}\right]=\frac{[n][n-1] \cdots[n-k+1]}{[k] !} .
$$

Proof For $0 \leq p \leq n$, let us write, more generally,

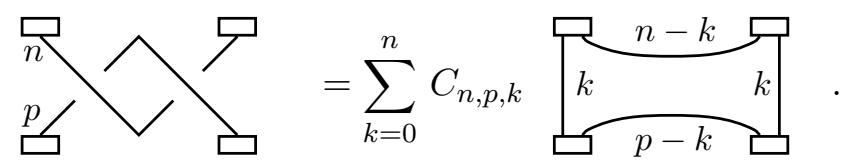

First, we consider the case $p=1$. By induction on $n$, it is easy to prove that

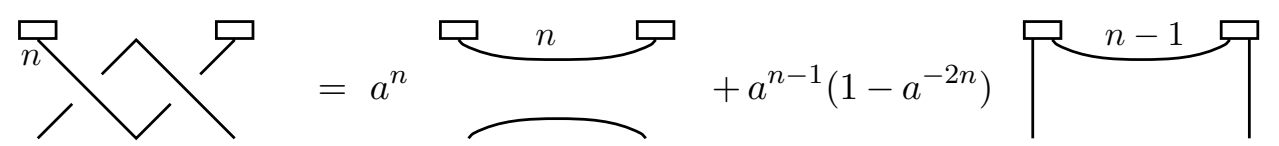

(recall $a=A^{2}$ ). Using this, we now fix $n$ and do induction on $p$ to obtain the recursion formula

$$
C_{n, p+1, k}=a^{n-2 k} C_{n, p, k}+a^{n-2 k+1}\left(1-a^{-2(n-k+1)}\right) C_{n, p, k-1} .
$$

Here we have used the following two facts which follow from the defining properties of the Jones-Wenzl idempotents. ${ }^{2}$

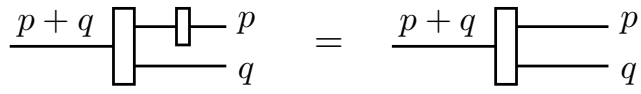

$$
\begin{aligned}
& \underline{p+q}<_{q}^{p}=A^{-p q} \quad \underline{p+q}{ }^{p}
\end{aligned}
$$

\footnotetext{
${ }^{2}$ Equation (39) is a special case of the half-twist coefficient.
} 
Note that the coefficients $C_{n, p, k}$ behave like the binomial coefficients $\left(\begin{array}{l}p \\ k\end{array}\right)$ in that $C_{n, 0,0}=1$, and $C_{n, p, k}=0$ for $k<0$ or $k>p$. It follows that the recursion formula (37) determines the $C_{n, p, k}$ uniquely. One finds

$$
C_{n, p, k}=a^{p(n-k)}\left[\begin{array}{l}
p \\
k
\end{array}\right] \prod_{j=n-k+1}^{n}\left(1-a^{-2 j}\right) .
$$

Specializing to the case $p=n$, this proves the Lemma.

Remark 4.2 The coefficient $C_{n, n}$ was computed by a different method in [A, Prop. 4.4]. Knowing this coefficient would be enough to obtain Habiro's formula (7) for $\omega$ (see Remark 4.4 below). Unfortunately, the method of [A] does not give the coefficients $C_{n, k}$ for $k \neq n$, which we will need to obtain a formula for $\omega^{p}$.

Lemma 4.3 We have

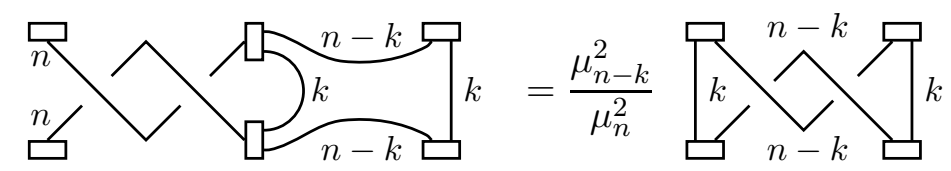

Proof The left hand side of (40) is equal to

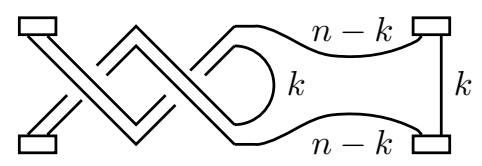

By an isotopy, this becomes

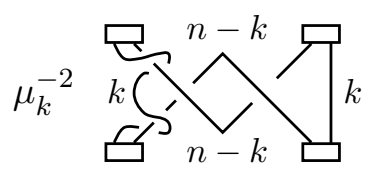

Applying the coefficients $\delta(n ; n-k, k)^{-2}$ and $\delta(n ; k, n-k)^{-2}$, which are both equal to $\mu_{k} \mu_{n-k} \mu_{n}^{-1}$ (see also (39)), we see that this is equal to the right hand side of (40). 
Let $C_{n, k}^{(p)}$ be the coefficient defined by the expansion

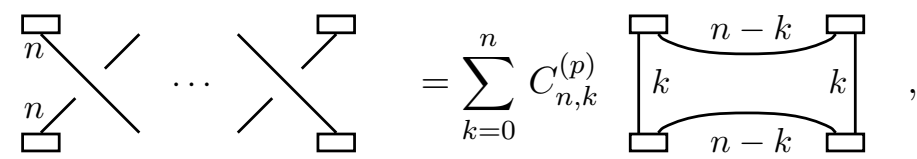

(where the diagram on the left hand side of (41) has $2 p$ crossings). Putting the two preceding Lemmas together, we may obtain a formula for this coefficient. In particular, it follows by induction on $p$ that $C_{n, k}^{(p)}$ is a Laurent polynomial divisible by $\prod_{j=n-k+1}^{n}\left(1-a^{2 j}\right)$.

We are interested in the coefficient $C_{n, n}^{(p)}$, since we have

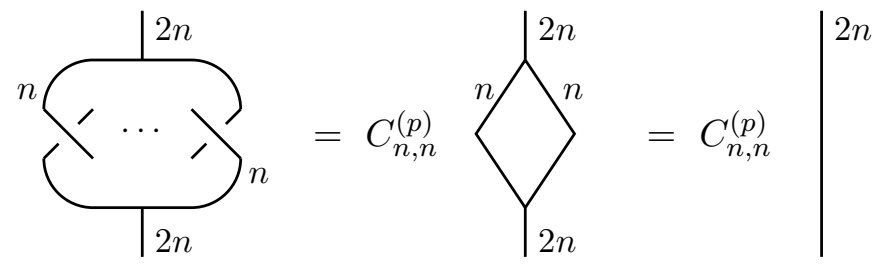

(where there are $2 p$ crossings in the diagram on the left). Using (30) and (31) from Section 3, it follows that

$$
\mu_{n}^{-2 p} c_{n, p}(-1)^{n}(\{n\} !)^{2}=C_{n, n}^{(p)}
$$

and therefore

$$
c_{n, p}^{\prime}=\{n\} ! c_{n, p}=(-1)^{n} \mu_{n}^{2 p}(\{n\} !)^{-1} C_{n, n}^{(p)} .
$$

As already observed, $C_{n, n}^{(p)}$ is divisible by

$$
\prod_{j=1}^{n}\left(1-a^{2 j}\right)=a^{-n(n+1) / 2}\{n\} !
$$

and hence $c_{n, p}^{\prime}$ is indeed a Laurent polynomial.

Remark 4.4 In the case $p=1$, we have

$$
C_{n, n}^{(1)}=C_{n, n}=a^{-n(n+1) / 2}\{n\} ! .
$$

Plugging this into (43), we get

$$
c_{n, 1}^{\prime}=(-1)^{n} a^{n^{2}+2 n} a^{-n(n+1) / 2}=(-1)^{n} a^{n(n+3) / 2},
$$

giving another proof of Habiro's formula (7) for $c_{n, 1}$. 
Here is an explicit formula for $C_{n, n}^{(p)}$ which follows from (36) and (40). The sum is over all multi-indices $\underline{k}=\left(k_{1}, \ldots, k_{p}\right)$ such that $k_{i} \geq 0$ for all $i$, and $\sum k_{i}=n$. For convenience, put $s_{i}=k_{1}+\ldots+k_{i}$ and $r_{i}=n-s_{i}=k_{i+1}+\ldots+k_{p}$, and define

$$
\begin{gathered}
\varphi(\underline{k})=\sum_{i=1}^{p-1} r_{i}\left(r_{i-1}+r_{i}+2\right) \\
C_{n, n}^{(p)}=\sum_{\underline{k}} C_{n, k_{1}} \frac{\mu_{n-k_{1}}^{2}}{\mu_{n}^{2}} C_{n-k_{1}, k_{2}} \frac{\mu_{n-k_{1}-k_{2}}^{2}}{\mu_{n}^{2}} \cdots C_{n-s_{p-2}, k_{p-1}} \frac{\mu_{n-s_{p-1}}^{2}}{\mu_{n}^{2}} C_{n-s_{p-1}, k_{p}} \\
=\mu_{n}^{2-2 p} \sum_{\underline{k}}\left(\prod_{i=1}^{p-1} \mu_{r_{i}}^{2}\right) \prod_{i=1}^{p} C_{r_{i-1}, k_{i}} \\
=\mu_{n}^{2-2 p}\left(\prod_{j=1}^{n}\left(1-a^{-2 j}\right)\right) \sum_{\underline{k}}\left(\prod_{i=1}^{p-1} a^{r_{i}{ }^{2}+2 r_{i}}\right) \prod_{i=1}^{p-1}\left(a^{r_{i-1} r_{i}}\left[\begin{array}{c}
r_{i-1} \\
k_{i}
\end{array}\right]\right) \\
=\mu_{n}^{2-2 p} a^{-n(n+1) / 2}\{n\} ! \sum_{\underline{k}} a^{\varphi(\underline{k})}\left[\begin{array}{c}
n \\
k
\end{array}\right]
\end{gathered}
$$

where we have put, as usual,

$$
\left[\begin{array}{l}
n \\
\underline{k}
\end{array}\right]=\frac{[n] !}{\left[k_{1}\right] ! \cdots\left[k_{p}\right] !} .
$$

In view of (43), and using $\mu_{n}^{2}=a^{n^{2}+2 n}$, we obtain the following final result:

Theorem 4.5 (Habiro [H3]) For $p \geq 1$, the coefficients $c_{n, p}^{\prime}$ of $\omega^{p}$ in (34) are given by

$$
c_{n, p}^{\prime}=(-1)^{n} a^{n(n+3) / 2} \sum_{\substack{k=\left(k, \ldots, k_{p}\right) \\
k_{i} \geq 0, \sum k_{i}=n}} a^{\varphi(\underline{k})}\left[\begin{array}{l}
n \\
\underline{k}
\end{array}\right] .
$$

where $\varphi(\underline{k})$ is defined in (45).

Remark 4.6 Since $c_{n,-p}^{\prime}=(-1)^{n} \overline{c_{n, p}^{\prime}}$, this also determines the coefficients of negative powers of $\omega$.

Remark 4.7 In [H3], Habiro has obtained a similar formula using the quantum group $U_{q} \mathfrak{s l}_{2}$. 
Example: Assume $p=2$. We may write $\underline{k}=(k, n-k)$. Then

$$
\begin{aligned}
c_{n, 2}^{\prime} & =(-1)^{n} a^{n(n+3) / 2} \sum_{k=0}^{n} a^{(n-k)(n+n-k+2)}\left[\begin{array}{l}
n \\
k
\end{array}\right] \\
& =(-1)^{n} a^{\left(5 n^{2}+7 n\right) / 2} \sum_{k=0}^{n} a^{k^{2}-2 k-3 n k}\left[\begin{array}{l}
n \\
k
\end{array}\right]
\end{aligned}
$$

\section{The colored Jones polynomial of twist knots}

In this last section, we illustrate one use of $\omega^{p}$, namely to give a formula for the colored Jones polynomial of twist knots (see Figure 3 ) in terms of the coefficients $c_{n, p}^{\prime}$. The results of this section are known to K. Habiro and T.Q.T. Le.

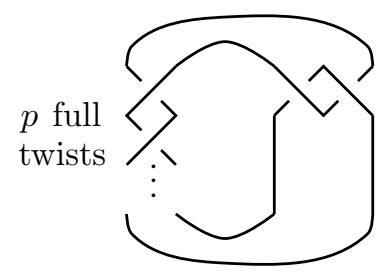

Figure 3: The twist knot $K_{p}$. (Here $p \in \mathbb{Z}$.) For $p=1, K_{p}$ is a left-handed trefoil, and for $p=-1, K_{p}$ is the figure eight knot.

The colored Jones polynomial of a knot $K$ colored with the $N$-dimensional irreducible representation of $\mathfrak{s l}_{2}$ can be expressed as the Kauffman bracket of $K$ cabled by $(-1)^{N-1} e_{N-1}$ :

$$
J_{K}(N)=(-1)^{N-1}\left\langle K\left(e_{N-1}\right)\right\rangle .
$$

(The factor of $(-1)^{N-1}$ is included so that $J_{U n k n o t}(N)=[N]$.) We will use the normalization

$$
J_{K}^{\prime}(N)=\frac{J_{K}(N)}{J_{U n k n o t}(N)}=\frac{\left\langle K\left(e_{N-1}\right)\right\rangle}{\left\langle e_{N-1}\right\rangle} .
$$

Here, we assume the knot $K$ is equipped with the zero framing.

Let us compute $\left\langle K_{p}\left(e_{N-1}\right)\right\rangle$. We use the surgery description given in Fig. 4. Recall that $\omega^{p}=\sum c_{k, p}^{\prime} R_{k}^{\prime}$. By induction, one can check that

$$
e_{N-1}=\sum_{n=0}^{N-1}(-1)^{N-1-n}\left[\begin{array}{c}
N+n \\
N-1-n
\end{array}\right] R_{n} \text {. }
$$


The key observation (which I learned from T.Q.T. Le) is that

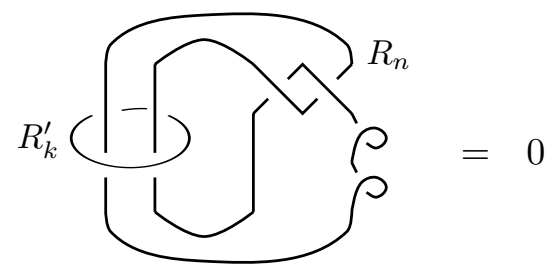

for $k \neq n$. This is because each component of this link is a zero-framed unknot having a spanning disk pierced twice by the other component, and circling with $R_{m}$ annihilates all even polynomials in $z$ of degree $<2 m$.

Thus only terms with $k=n$ survive, and so we have

$$
\left\langle K_{p}\left(e_{N-1}\right)\right\rangle=\sum_{n=0}^{N-1}(-1)^{N-1-n}\left[\begin{array}{c}
N+n \\
N-1-n
\end{array}\right] c_{n, p}^{\prime}
$$

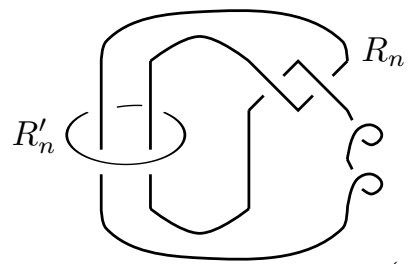

Now, using that $R_{n}-e_{n}$ has degree $<n$, we compute

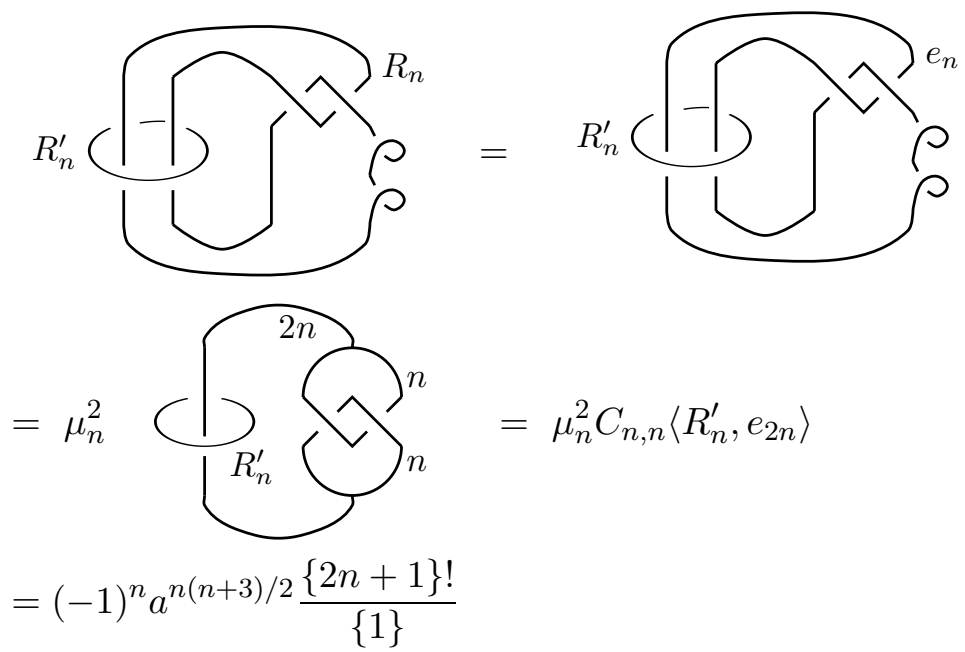

where we have used (42) in the last but one equation, and $C_{n, n}$ and $\left\langle R_{n}, e_{2 n}\right\rangle$ are given in (44) and (5), respectively (but notice we are using $R_{n}^{\prime}=(\{n\} !)^{-1} R_{n}$ here).

Plugging this into (48), we obtain the following result.

Algebraic 83 Geometric Topology, Volume 3 (2003) 


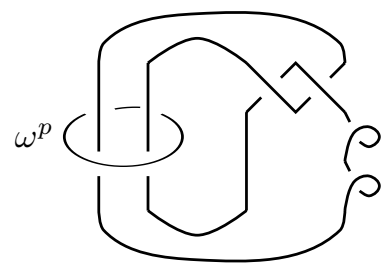

Figure 4: A surgery description of the twist knot $K_{p}$ with zero framing.

Theorem 5.1 The colored Jones polynomial of the twist knot $K_{p}$ is given by

$$
J_{K_{p}}^{\prime}(N)=\sum_{n=0}^{\infty} f_{K_{p}, n} \frac{\{N-n\}\{N-n+1\} \cdots\{N+n\}}{\{N\}},
$$

where

$$
f_{K_{p}, n}=a^{n(n+3) / 2} c_{n, p}^{\prime} .
$$

(The sum is actually finite, the terms with $n \geq N$ being all zero.)

Note that since $c_{n, p}^{\prime}$ is a Laurent polynomial in $a$, so are the coefficients $f_{K_{p}, n}$. For example, for the figure eight knot $K_{-1}$, we have $f_{K_{-1}, n}=1$, and for the lefthanded trefoil $K_{1}$, we have $f_{K_{1}, n}=(-1)^{n} a^{n(n+3)}$. (The right-handed trefoil is the mirror image of $K_{1}$, so one just needs to take the conjugate of $J_{K_{1}}^{\prime}(N)$.) These formulas can be found in [H2].

Remark 5.2 Here is another expression for $J_{K_{p}}^{\prime}(N)$. Put $q=a^{2}=A^{4}$ and, as is customary in $q$-calculus,

$$
(x)_{n}=(1-x)(1-x q) \cdots\left(1-x q^{n-1}\right) .
$$

Then (49) gives

$$
J_{K_{p}}^{\prime}(N)=\sum_{n=0}^{\infty} \tilde{f}_{K_{p}, n}\left(q^{1-N}\right)_{n}\left(q^{1+N}\right)_{n}
$$

where

$$
\tilde{f}_{K_{p}, n}=(-1)^{n} q^{-n(n+1) / 2} f_{K_{p}, n} .
$$

For example, for the figure eight knot $K_{-1}$, we have $\tilde{f}_{K_{-1}, n}=(-1)^{n} q^{-n(n+1) / 2}$, and for the left-handed trefoil $K_{1}$, we have $\tilde{f}_{K_{1}, n}=q^{n}$. These formulas can be found in [L2] (see also [L1]). 


\section{References}

[A] H. ABChIR. TQFT invariants at infinity for the Whitehead manifold. In: Knots in Hellas '98, Proceedings of the International Conference on Knot Theory and Its Ramifications, World Scientific, Series on Knots and Everything, Vol. 24.

[BHMV1] C. Blanchet, N. Habegger, G. Masbaum, P. Vogel. Threemanifold invariants derived from the Kauffman bracket, Topology 31 (1992), $685-699$

[BhmV2] C. Blanchet, N. Habegger, G. Masbaum, P. Vogel. Topological quantum field theories derived from the Kauffman bracket, Topology 34 (1995), 883-927

[H1] K. HABIRo. On the colored Jones polynomial of some simple links. In: Recent Progress Towards the Volume Conjecture, Research Institute for Mathematical Sciences (RIMS) Kokyuroku 1172, September 2000.

[H2] K. HABIRO. On the quantum $s l_{2}$ invariants of knots and integral homology spheres. In: Invariants of knots and 3-manifolds (Kyoto 2001), Geometry and Topology Monographs, Vol. 4 (2002), 55-68.

[H3] K. HaBIRO. In preparation.

[Ka] R.M. Kashaev. The hyperbolic volume of knots from quantum dilogarithm. Lett. Math. Phys. 39 (1997) 269-275.

[KL] L. H. Kauffman, S. L. Lins. Temperley-Lieb recoupling theory and invariants of 3-manifolds, Ann. Math. Studies 133 (Princeton University Press, 1994).

[L1] T.Q.T. LE. Quantum invariants of 3-manifolds: integrality, splitting, and perturbative expansion. arXiv:math.QA/0004099

[L2] T.Q.T. LE. In preparation.

[MV] G. Masbaum, P. Vogel. 3-valent graphs and the Kauffman bracket. Pacific J. Math. 164, (1994) 361-381.

[MM] H. Murakami, J. Murakami. The colored Jones polynomial and the simplicial volume of a knot. Acta Math. 186 (2001) 85-104.

Institut de Mathématiques de Jussieu (UMR 7586 du CNRS)

Université Paris 7 (Denis Diderot), Case 7012

2, place Jussieu, 75251 Paris Cedex 05, France

Email: masbaum@math.jussieu.fr

Received: 12 December 2002 